Tabelle 6. Umfang dev Zuchtstamm-, Vor- und Hauptprïfung 1963.

\begin{tabular}{|c|c|c|c|c|c|c|}
\hline \multirow{2}{*}{$\begin{array}{c}\text { Jahrgang } \\
\text { der Stämme }\end{array}$} & \multirow{2}{*}{ Prüfung } & \multirow{2}{*}{ Reifegruppe } & \multirow{2}{*}{$\begin{array}{l}\text { Anzahl insgesamt } \\
\text { (Lüsewitz und } \\
\text { Außenstellen) }\end{array}$} & \multicolumn{3}{|c|}{ davon } \\
\hline & & & & $\mathrm{N}$ & B & $\mathrm{N}+\mathrm{G}$ \\
\hline 1960 & $\mathrm{ZV} \mathrm{I}$ & $\begin{array}{l}\text { fr: } \\
\text { mfr. } \\
\text { msp. } \\
\text { sp. }\end{array}$ & $\begin{array}{r}136 \\
147 \\
258 \\
55\end{array}$ & $\begin{array}{l}11 \\
28 \\
32 \\
13\end{array}$ & $\begin{array}{l}1 \\
2 \\
4 \\
1\end{array}$ & \\
\hline 1959 & $Z I$ & $\begin{array}{l}\text { insges. } \\
\text { fr. } \\
\text { mfr. } \\
\text { msp. } \\
\text { sp. }\end{array}$ & $\begin{array}{r}596 \\
\\
41 \\
68 \\
86 \\
-10\end{array}$ & $\begin{array}{r}1 \\
3 \\
12 \\
2\end{array}$ & $\begin{array}{l}2 \\
9 \\
1\end{array}$ & 1 \\
\hline $195^{8}$ & $Z$ II & $\begin{array}{l}\text { insges. } \\
\text { fr. } \\
\text { mfr. } \\
\text { msp. } \\
\text { sp. }\end{array}$ & $\begin{array}{l}205 \\
17 \\
31 \\
29 \\
12\end{array}$ & $\begin{array}{l}6 \\
7 \\
3\end{array}$ & $\begin{array}{l}3 \\
1 \\
2\end{array}$ & $\begin{array}{l}1 \\
3\end{array}$ \\
\hline 1957 & VP I & $\begin{array}{l}\text { insges. } \\
\text { fr. } \\
\text { mfr. } \\
\text { msp. } \\
\text { sp. }\end{array}$ & $\begin{array}{l}89 \\
7 \\
5 \\
8 \\
2\end{array}$ & $\begin{array}{l}1 \\
1 \\
1\end{array}$ & $\begin{array}{l}1 \\
2 \\
2\end{array}$ & \\
\hline $195^{6}$ & VP II & $\begin{array}{l}\text { insges. } \\
\text { fr. } \\
\text { mfr. } \\
\text { msp. } \\
\text { sp. }\end{array}$ & $\begin{array}{r}22 \\
6 \\
2 \\
4 \\
2\end{array}$ & 1 & 3 & \\
\hline 1955 & HP I & $\begin{array}{l}\text { insges. } \\
\text { fr. } \\
\text { mfr. } \\
\text { msp. } \\
\text { sp. }\end{array}$ & $\begin{array}{l}14 \\
3 \\
4 \\
1 \\
1\end{array}$ & & 2 & \\
\hline 1954 & $\mathrm{HP} \mathrm{II}$ & $\begin{array}{l}\text { insges. } \\
\text { fr. } \\
\text { mfr. } \\
\text { msp. } \\
\text { sp. }\end{array}$ & $\begin{array}{l}9 \\
1 \\
1 \\
1\end{array}$ & & & \\
\hline & & insges. & 2 & & & \\
\hline
\end{tabular}

$\mathrm{N}=$ Nematodenresistenz; $\mathrm{G} \mathrm{bzw}: \mathrm{B}=$ Krebsbiotypenresistenz.

gewonnenen Versuchsergebnisse. Die Prüfungsstationen unterstehen der Abteilung Agrotechnik, Stammesprüfung, Veredlung und Vermarktung des Institutes. Die Züichter können dadurch auf manche der früher erforderlichen eigenen Leistungsprüfungen verzichten und ihre Aufmerksamkeit der Anwen- dung einfacherer, wenig aufwendiger Selektionsverfahren zuwenden. Die meisten Kartoffelzüchter der DDR haben bereits die eigenen Leistungsprüfungen eingestellt. Es ist sehr günstig, daß die Stämme so. wohl im Vergleich zu den Standardsorten als auch zu den Stämmen der verschiedenen Züchter geprüft werden.

Mehrere Auslandsreisen und Literaturstudium lassen uns zu dem Schluß gelangen, daß eine derart intensive und frühzeitig einsetzende Zuchtstammprüfung in anderen Ländern z. $Z$. noch nicht üblich ist (s. RehSe, 1961, Geyer, 1961, ANONYM, 1962).

\section{Zusammenfassung}

1. Es wurde an Hand des Prüfungsschemas der Kartoffelzuchtstämme in der DDR der Prüfungsgang im einzelnen erläutert. Bei der Besprechung der Feldversuche wurde auch auf Methodik und Umfang eingegangen und dargelegt, welche Ergebnisse bei der Virusresistenzzüchtung und auf einigen anderen Gebieten erzielt wurden.

2. Die Prüfung der Stämme aller Züchter beginnt bereits mit Material aus der Ernte der A-Klone auf Krebs- und Nematodenresistenz, aus der Ernte der B-Klone im Feldversuch auf Virusresistenz und aus der Ernte der C-Klone an 5-6 Orten auf Ertrag und Qualitätseigenschaften.

3. Die Züchter können auf den größten Teil der eigenen Leistungsprüfungen verzichten, weil sie für eine Vielzahl von Merkmalen repräsentative und sichere Ergebnisse erhalten.

4. Durch die Zuchtstammprüfungen wird erreicht, $\mathrm{da} \beta$ in die staatlichen Prüfungen nur noch gute Zuchtstämme, die ihre hohe Leistungsfähigkeit unter Beweis gestellt haben, aufgenommen werden.

\section{Literatur}

1. Anonym: Biul. Inst. Hodowli i Aklimat. Roslin Nr. $2-3,43-46$ (1962). - 2. GaLL, H.: Untersuchungen zur Auswertung langjähriger Sortenversuche bei Kartoffeln. Z. f. landw. Versuchs- u. Untersuchungswesen 3, 319 ff. (1957) . - 3. Geyer, H.: Deutsche Pflanzkartoffeln sichern hohe Ernten im Kartoffelbau. Saatgutwirtsch., Sonderh., S. 15-17 (1961). - 4. PfEFFer, CH.: Untersuchungen über den wert der in verschiedenen Gebieten erzeugten Pflanzkartoffeln. Der Züchter 26, 257-269 (1956). - 5. Rerse, E.: Grundzüge der Organisation der Pflanzenzüchtung und des Saatgutwesens in den Niederlanden. Schriftenr. landw. Fak. Univ. Kiel Nr. 29, 1-63 (1961), - 6. Schick, R, u. M. KLINKowski: Die Kartoffel - ein Handbuch. Bd. II, S. $1476-1483$. Berlin: Deutscher Landwirtschaftsverlag 1962 .

\title{
KURZE MITTEILUNG
}

\section{Tagungen der Arbeitsgemeinschaft Getreideforschung 1964}

Die Arbeitsgemeinschaft Getreideforschung beabsichtigt, im 2. Halbjahr 1964 folgende Tagungen durchzuführen:
1. Tagung für Müllereitechnologie 7.-9. 10. 1964 2. Tagung für Bäckereitechnologie 20.-22. 10. 1964

Beide Tagungen finden in Detmold, im RoemerHaus der Arbeitsgemeinschaft Getreideforschung statt. 